\title{
INDEX THEORY FOR LINEAR ELLIPTIC EQUATION \\ AND MULTIPLE SOLUTIONS \\ FOR ASYMPTOTICALLY LINEAR ELLIPTIC EQUATION WITH RESONANCE
}

\author{
YUAN SHAN - KEQIANG LI
}

\begin{abstract}
In this paper, we consider the existence and multiplicity of solutions to the elliptic equation with resonance. We classify the linear elliptic equation and obtain some new conditions on the existence and multiplicity for asymptotically linear elliptic equation by using critical point theory.
\end{abstract}

\section{Introduction and main results}

In this paper, we consider the following problem:

$$
\begin{aligned}
\Delta u+f(x, u)+f_{1}(x, u) & =0, \\
\left.u\right|_{\partial \Omega} & =0,
\end{aligned}
$$

where $\Omega \subset \mathbb{R}^{n}$ is a bounded open domain, with smooth boundary $\partial \Omega, \Delta u=$ $\sum_{i=1}^{n} \frac{\partial^{2}}{\partial x_{i}^{2}} u$ and $f: \bar{\Omega} \times \mathbb{R} \rightarrow \mathbb{R}$ is a $L^{1}$-Carathéodory function, i.e. $f(x, \cdot)$ is continuous on $\mathbb{R}$ for almost every $x \in \bar{\Omega}, f(\cdot, u)$ is measurable on $\Omega$ for each $u \in \mathbb{R}$. Moreover, for any constant $r>0$, there exists some function $\rho_{r} \in L^{1}(\Omega)$ such

2010 Mathematics Subject Classification. 58E05, 58E50.

Key words and phrases. Index theory, elliptic equation, asymptotically linear conditions, critical point theory, multiple solutions.

Partially supported by the Jiangsu Planned Projects for Postdoctoral Research Funds (1302012B) and the NSF of China (Tianyuan Fund for Mathematics, 11326134). 
that $|f(x, u)| \leq \rho_{r}(x)$ for almost every $x \in \Omega$ and all $u \in \mathbb{R}$ with $|u| \leq r$. And $f_{1}: \bar{\Omega} \times \mathbb{R} \rightarrow \mathbb{R}$ is also a Carathéodory function such that

$$
\left|f_{1}(x, u)\right| \leq \widetilde{g}(t)
$$

for $x \in \mathbb{R}$ and almost every $t \in \Omega$, where $\widetilde{g} \in L^{2}(0,1)$. We look for the weak solutions of (1.1) and (1.2) which are the same as the critical points of the functional

$$
I(u)=\frac{1}{2} \int_{\Omega}|\nabla u|^{2} d x-\int_{\Omega} F(x, u) d x-\int_{\Omega} F_{1}(x, u) d x,
$$

for all $u \in H_{0}^{1}(\Omega)$, where

$$
F(x, u)=\int_{0}^{u} f(x, t) d t, \quad F_{1}(x, u)=\int_{0}^{u} f_{1}(x, t) d t
$$

and $H_{0}^{1}(\Omega)$ is the Sobolev space with the norm

$$
\|u\|=\|\nabla u\|_{2}=\left(\int_{\Omega}|\nabla u|^{2} d x\right)^{1 / 2} .
$$

Many results on the existence of solutions for Duffing equation and elliptic equation satisfy asymptotically linear condition. For the non-resonant nonlinearities, we refer to [6], [16]. For the resonant nonlinearities, we refer to [10]-[13], [17], [18]. It is remarkable that there are great deal of approaches for proving these results, including topological degree technique, the critical point theory and the Morse theory. However, there are few results on the multiple solutions to equations with resonance.

We assume that $f$ is asymptotically linear at infinity: there exist functions $a, b \in L^{\infty}(\Omega)$ such that

$$
\left(\mathrm{f}_{1}\right) a(x) \leq \frac{f(x, u)}{u} \leq b(x), \quad \text { for all } x \in \Omega,|u|>r>0 .
$$

It should be mentioned here that this type condition has been widely considered in Hamiltonian systems. A quantitative way to measure the asymptotically linear condition is the index theory. As in [6], an index for the second order linear Hamiltonian systems was defined. And in [7], an index theory for first order linear Hamiltonian systems was developed. In [4], [14], [15], by Conley, Zehnder and Long, an index theory for symplectic path was defined.

In [8], an index theory was established to investigate the self-adjoint operator equation with finite Morse index which can also be used to study second-order Hamiltonian system and elliptic equation. Since the functional defined in (1.3) is bounded from below, we establish the classification theory for the associated linear elliptic equation of (1.1) following the ideas of [6] and [8]. This paper is largely motivated by the work of [10] in which the existence of solutions to a semilinear elliptic equation with double resonance was proved. 
In this paper, we also consider problem (1.1) and (1.2) with resonance under the asymptotically linear condition $\left(\mathrm{f}_{1}\right)$. Obviously, $(1.1)$ does not satisfy the linear growth condition: there exists a constant $C_{0}>0$ such that

$$
|f(x, u)| \leq C_{0}(1+|u|), \quad \text { for all } u \in \mathbb{R}, x \in \Omega .
$$

In order to obtain better results than the earlier works, an index theory for associated linear elliptic equations needs to be developed. Since the functional defined in (1.3) is bounded from below, the index theory for linear elliptic equation will be established by using Morse index. Thanks to this index theory, the existence and multiplicity of critical points of (1.3) were established. And our main results are obtained for the case when $I$ is even.

For any $b(x) \in L^{\infty}(\Omega)$, we consider the following linear elliptic equation:

$$
\Delta u+b(x) u=0,\left.\quad u\right|_{\partial \Omega}=0 .
$$

Set $E=H_{0}^{1}(\Omega)$. Define

$$
q_{b}(u, v)=\int_{\Omega}(\nabla u(x), \nabla v(x)) d x-\int_{\Omega}(b(x) u(x), v(x)) d x,
$$

for all $u, v \in E$.

Definition 1.1 ([8, Definition 2.5.1]). For any $b \in L^{\infty}(\Omega)$, we define

$$
\begin{aligned}
\nu(b) & =\operatorname{dim} \operatorname{ker}\left(q_{b}\right)=\operatorname{dim}\left\{u \mid q_{b}(u, v)=0, \text { for all } v \in E\right\}, \\
i(b) & =\sum_{\lambda<0} \nu(b+\lambda) .
\end{aligned}
$$

We call $i(b)$ the index and $\nu(b)$ the nullity of $b$, respectively.

For any $q \in L^{\infty}(\Omega)$, let $\lambda_{1}(q) \leq \lambda_{2}(q) \leq \ldots \leq \lambda_{k}(q) \leq \ldots$ be the eigenvalues of (1.2) and

$$
-\Delta u-q(x) u=\lambda u .
$$

For any $a, b \in L^{\infty}(\Omega)$, which satisfy $\left(\mathrm{f}_{1}\right)$, the non-resonant condition

$$
\lambda_{k}(a)<0, \quad \lambda_{k+1}(b)>0
$$

can be rewritten as

$$
i(a)=i(b)=k, \quad \nu(b)=0,
$$

and the double-resonance condition

$$
\lambda_{k}(a) \leq 0, \quad \lambda_{k+1}(b) \geq 0
$$

can be rewritten as

$$
i(a)+\nu(a)=i(b)=k .
$$

We will express this in Proposition 2.2.4 after necessary discussion on the property of the index. 
Throughout this paper, for any $a_{1}, a_{2} \in L^{\infty}(\Omega)$, we write $a_{1} \leq a_{2}$, if $a_{2}(x)-$ $a_{1}(x) \geq 0$, for almost every $x \in \Omega$; we write $a_{1}<a_{2}$, if $a_{1} \leq a_{2}$, and $a_{2}(x)-$ $a_{1}(x)>0$ holds on a subset of $[0,1]$ with nonzero measure. In condition $\left(\mathrm{f}_{1}\right)$, without loss of generality, we assume that $\Omega_{0}=\{x \in \Omega \mid a(x) \neq b(x)\}$ is not empty. Thus, by definition, we have $a<b$. Now we use the index to reach our main results.

THEOREM 1.2. Suppose that condition $\left(\mathrm{f}_{1}\right)$, condition:

$\left(\mathrm{f}_{2}\right) i(b)=i(a)+\nu(a)$,

and the following generalized Landesman-Lazer conditions:

$\left(\mathrm{f}_{3}^{+}\right) \lim _{\|v\| \rightarrow \infty} \int_{\Omega}\left[F(x, v(x))+F_{1}(x, v(x))-\frac{a(x)}{2} v^{2}(x)\right] d x=+\infty, v \in \operatorname{ker}\left(q_{a}\right)$,
$\left(\mathrm{f}_{3}^{-}\right) \lim _{\|v\| \rightarrow \infty} \int_{\Omega}\left[F(x, v(x))+F_{1}(x, v(x))-\frac{b(x)}{2} v^{2}(x)\right] d x=-\infty, v \in \operatorname{ker}\left(q_{b}\right)$, are satisfied. Then problem (1.1)-(1.2) has at least one weak solution for $n \leq 4$.

Corollary 1.3. Under the assumptions of Theorem 1.2, if $f_{1}(x, u)=0$, problem (1.1)-(1.2) has at least one weak solution for any $n \in \mathbb{N}$.

REMARK 1.4. (a) Due to the Sobolev inequality

$$
\|u\|_{L^{q}} \leq C(n, p)\|u\|_{w^{1, p}},
$$

with $q=n p /(n-p)$, we restrict $n \leq 4$, when $f_{1} \neq 0$.

(b) Note that Corollary 1.3 is similar to Theorem 1.1 in [10], but for different $a$ and $b$. In this paper we consider a more general form of $a$, instead of $a$ is a continuous function in [10], we assume that $a \in L^{\infty}(\Omega)$. In this sense, our result extends the result in [10].

EXAmple 1.5. Suppose that $n=1, \Omega=(0,1)$. Let $t_{1} \in(0,1)$ be fixed,

$$
a(x)=\left\{\begin{array}{ll}
\frac{\pi^{2}}{4 t_{1}^{2}} & \text { if } x \in\left(0, t_{1}\right), \\
\frac{\pi^{2}}{4\left(1-t_{1}\right)^{2}} & \text { if } x \in\left(t_{1}, 1\right) .
\end{array} \quad h(x, u)= \begin{cases}u e^{-u}+\sin x & \text { if } u<0, \\
\arctan u+\sin x & \text { if } u>0 .\end{cases}\right.
$$

The following problem

$$
u^{\prime \prime}+a(x) u+h(x, u)=0, \quad u(0)=0=u(1)
$$

has at least one solution. In fact, let

$$
\begin{array}{ll}
u_{0}(t)=\sin \frac{\pi}{2 t_{1}} t & \text { for } t \in\left(0, t_{1}\right), \\
u_{0}(t)=\sin \left(\frac{\pi}{2\left(1-t_{1}\right)}\left(t-t_{1}\right)+\frac{\pi}{2}\right) & \text { for } t \in\left(t_{1}, 1\right) .
\end{array}
$$

Then $u=u_{0}(t)$ is a nontrivial solution of

$$
u^{\prime \prime}+a(x) u=0, \quad u(0)=0=u(1),
$$


and by Definition 1.1, we have $i(a)=0, \nu(a)=1$ and $\operatorname{ker}\left(q_{a}\right)=\left\{c u_{0} \mid c \in \mathbb{R}\right\}$. All the assumptions of Theorem 1.2 are satisfied. Hence, problem (1.1)-(1.2) has at least one solution. Especially, $a(x)$ is a continuous function if and only if $t_{1}=1 / 2$.

THEOREM 1.6. Under the assumptions of Theorem 1.2, for $n<4$, if further, $f(-x)=-f(x), f(0)=\theta$ and $f$ satisfies following condition:

$\left(\mathrm{f}_{4}\right)$ there exists $A(x, u) \in C(\Omega \times \mathbb{R}, \mathbb{R})$ such that

$$
\begin{aligned}
f(x, u)+f_{1}(x, u) & =A(x, u) u+o(|u|) \quad \text { as }|u| \rightarrow 0, \\
A(x, u) & \leq a_{1}(x),
\end{aligned}
$$

with $i(a)-\nu(a)>i\left(a_{1}\right)+\nu\left(a_{1}\right)$, then (1.1)-(1.2) have at least $i(a)-$ $i\left(a_{1}\right)-\nu(a)-\nu\left(a_{1}\right)$ pairs of weak solutions.

Corollary 1.7. Under the assumptions of Corollary 1.3, if $f_{1}(x, u)=0$, for any $n \in \mathbb{N}$, we have that problem (1.1)-(1.2) has at least $i(a)-i\left(a_{1}\right)-\nu(a)-\nu\left(a_{1}\right)$ pairs of weak solutions.

From these two corollaries, our paper can be regarded as the further work and generalization of [10].

This paper is organized as follows: In Section 2, we introduce some preliminaries including the index theory and some theorems about critical point theory that we need in the proofs. In Section 3, we will prove Theorems 1.2 and 1.6.

\section{Index theory for linear elliptic equation}

In this section, we first decompose the space $E$ by $q_{b}$ which is defined in (1.5).

For any $u, v \in E$ if $q_{b}(u, v)=0$, we say that $u$ and $v$ are $q_{b}$-orthogonal. For any two subspaces $E_{1}$ and $E_{2}$ of $E$, if $q_{b}(u, v)=0$, for any $u \in E_{1}, v \in E_{2}$, we say that $E_{1}$ and $E_{2}$ are $q_{b}$-orthogonal.

Proposition 2.1. For any $b \in L^{\infty}(\Omega)$, the space $E$ has a $q_{b}$-orthogonal decomposition

$$
E=E^{+}(b) \oplus E^{0}(b) \oplus E^{-}(b),
$$

such that $q_{b}$ is positive definite, zero and negative definite on $E^{+}(b), E^{0}(b)$ and $E^{-}(b)$, respectively. Moreover, $E^{0}(b)$ and $E^{-}(b)$ are finitely dimensional.

REMARK 2.2. The decomposition was first used in [6] to investigate second order Hamiltonian system. This was further used in studying self-adjoint operator equation with finite Morse index in [8]. We will present the proof that we needed in the study of the properties of index and the expression of resonant conditions. 
Proof. Let $\lambda_{0}$ be a positive number satisfying $\lambda_{0}>b$. The norm $\|\cdot\|_{\lambda_{0}}$ defined by an product

$$
(u, v)_{\lambda_{0}}=\int_{\Omega}(\nabla u(x), \nabla v(x)) d x+\int_{\Omega}\left(\left(\lambda_{0}-b(x)\right) u(x), v(x)\right) d x,
$$

for all $u, v \in E$, is equivalent to $\|\cdot\|$ and $\left(E,(\cdot, \cdot)_{\lambda_{0}}\right)$ is a new Hilbert space. Let $K_{\lambda_{0}}(b): E \rightarrow E$ be an operator defined by

$$
\int_{\Omega}(u(x), v(x)) d x=\left(u, K_{\lambda_{0}}(b) v\right)_{\lambda_{0}}, \quad \text { for all } u, v \in E .
$$

Here we use the Riesz representation theorem. Since $E \hookrightarrow L^{2}$ is compact embedding, $K_{\lambda_{0}}$ is self-adjoint and compact. By the spectral theory of self-adjoint and compact operators, there exists a basis $\left\{e_{j}\right\} \subset E$ and $\mu_{j}(b) \rightarrow 0$ in $\mathbb{R}$ such that

$$
\left(e_{i}, e_{j}\right)_{\lambda_{0}}=\delta_{i j}, \quad K_{\lambda_{0}} e_{j}=\mu_{j}(b) e_{j},
$$

and $\mu_{j}(b)=\left(e_{j}, K_{\lambda_{0}} e_{j}\right)_{\lambda_{0}}=\left\|e_{j}\right\|_{2}^{2}>0$.

For any $x \in \sum_{j=1}^{\infty} c_{j} e_{j}$, from (2.1)-(2.3), a simple calculation shows that

$$
q_{b}(u, u)=(u, u)_{\lambda_{0}}-\lambda_{0}\|u\|_{2}^{2}=\sum_{j=1}^{\infty}\left(1-\lambda_{0} \mu_{j}(b)\right) c_{j}^{2}=\sum_{j=1}^{\infty}\left(\frac{1}{\mu_{j}(b)}-\lambda_{0}\right) c_{j}^{2}\left\|e_{j}\right\|_{2}^{2} .
$$

Set $\lambda_{j}(b)=1 / \mu_{j}(b)-\lambda_{0}$. From $\mu_{j} \rightarrow 0$ and $\mu_{j}>0$, we have that $\lambda_{j}(b) \rightarrow+\infty$ are positive except a finite number. Without loss of generality, we assume that $\lambda_{1}(b) \leq \ldots \leq \lambda_{k}(b) \leq \ldots$ Here the result holds, if we set

$$
\begin{aligned}
& E^{-}(b):=\left\{\sum_{j=1}^{\infty} \xi_{j} e_{j} \mid \xi_{j}=0 \text { if } \lambda_{j}(b) \geq 0\right\}, \\
& E^{0}(b):=\left\{\sum_{j=1}^{\infty} \xi_{j} e_{j} \mid \xi_{j}=0 \text { if } \lambda_{j}(b) \neq 0\right\}, \\
& E^{+}(b):=\left\{\sum_{j=1}^{\infty} \xi_{j} e_{j} \mid \xi_{j}=0 \text { if } \lambda_{j}(b) \leq 0\right\} .
\end{aligned}
$$

REMARK 2.3. (a) The above decomposition $E=E^{+}(b) \oplus E^{0}(b) \oplus E^{-}(b)$ is orthogonal with respect to $(\cdot, \cdot)_{2}$. To show the $(\cdot, \cdot)_{2}$ orthogonality, observe that, take $e_{i} \in E^{+}, e_{j} \in E^{-}$for example

$$
\left(e_{i}, e_{j}\right)_{2}=\left(e_{i}, K_{\lambda_{0}}(b) e_{j}\right)_{\lambda_{0}}=\lambda_{j}\left(e_{i}, e_{j}\right)_{\lambda_{0}}=0 .
$$

(b) The subspace $\operatorname{Ker}\left(q_{a}\right)$ and $\operatorname{Ker}\left(q_{b}\right)$ in condition $\left(f_{3}^{ \pm}\right)$can be expressed as $E^{0}(a)$ and $E^{0}(b)$, respectively.

Definition 2.4. For any $b \in L^{\infty}(\Omega)$, we define $i(b)=\operatorname{dim} E^{-}(b), \nu(b)=$ $\operatorname{dim} E^{0}(b)$. We call $i(b)$ and $\nu(b)$ the index and the nullity of $b$, respectively. 
Recall that for a bounded symmetric bilinear form $\phi$ defined on a Hilbert space $X$, its Morse index and nullity are defined as $m^{-}(\phi)=\max \left\{\operatorname{dim} X_{1} \mid\right.$ $X_{1}$ is a subspace of $\mathrm{X}$ such that $\phi(x, x)<0$, for any $\left.x \in X_{1} \backslash\{\theta\}\right\}$ and $m^{0}(\phi)=$ $\operatorname{dim} \operatorname{ker} \phi$ where $\operatorname{ker} \phi=\{x \in X \mid \phi(x, y)=0$, for all $y \in X\}$.

Applying [8, Proposition 2.1.3, Definition 2.5.1, Proposition 2.5.2], one can prove

Proposition 2.5. For any $b \in L^{\infty}(\Omega)$, we have:

(a) $i(b)=\sum_{\lambda_{i}(b)<0} \operatorname{dim} E_{i}(b)=\sum_{\lambda<0} \nu(b+\lambda)$, where $\lambda_{i}(b)$ is defined as in Proposition 2.1. Moreover, $i(b)=\sharp\left\{i \mid \lambda_{i}(b)<0\right\}, \nu(b)=\sharp\left\{i \mid \lambda_{i}(b)=0\right\}$, where ${ }^{\sharp} S$ denotes the total number of elements in a set $S$.

(b) $i(b)$ is the Morse index of $q_{b}$.

(c) If $b_{1} \leq b_{2}$, then

$$
i\left(b_{2}\right)-i\left(b_{1}\right)=\sum_{\lambda \in[0,1)} \nu\left(b_{1}+\lambda\left(b_{2}-b_{1}\right)\right) .
$$

The summand denoted by $I\left(b_{1}, b_{2}\right)$ is called the relative Morse index between $b_{1}$ and $b_{2}$.

(d) For any $b_{1}, b_{2} \in L^{\infty}(\Omega)$, if $b_{1} \leq b_{2}$, we have $i\left(b_{1}\right) \leq i\left(b_{2}\right)$; if $b_{1}<b_{2}$, we have $i\left(b_{1}\right)+\nu\left(b_{1}\right) \leq i\left(b_{2}\right)$.

(e) There exists $\varepsilon_{0}>0$ such that for any $\varepsilon \in\left(0, \varepsilon_{0}\right]$, we have

$$
\nu(b+\varepsilon)=0=\nu(b-\varepsilon), \quad i(b-\varepsilon)=i(b), \quad i(b+\varepsilon)=i(b)+\nu(b) .
$$

Proposition 2.6. For any $a, b \in L^{\infty}(\Omega)$ and $a<b$ :

(a) $\lambda_{k}(a)<0$, and $\lambda_{k+1}(b)>0$ if and only if $i(a)=i(b)=k$ and $\nu(b)=0$.

(b) $\lambda_{k}(a) \leq 0$, and $\lambda_{k+1}(b) \geq 0$ if and only if $i(a)+\nu(a)=i(b)=k$.

(c) $\lambda_{k}(a)=0$, and $\lambda_{k+1}(b)=0$ if and only if $i(a)+\nu(a)=i(b)=k$ and $\nu(b) \neq 0, \nu(a) \neq 0$.

Proof. (a) From Proposition 2.5(a), we have $i(a) \geq k, i(b) \leq k$. By Proposition 2.5(d), since $a<b$ we have $i(a) \leq i(a)+\nu(a) \leq i(b)$. Hence $i(a)=i(b)=k$ and $\lambda_{k}(b)<0$. From the monotonicity of $\lambda_{i}(b)$ with respect to $i$, one has $\lambda_{i}(b) \neq 0$ for all $i$. So $\nu(b)=0$.

(b) First, $\lambda_{k+1}(b) \geq 0$, we have $i(b) \leq k$. Since $a<b$, we have $i(a) \leq k$ and $\lambda_{k+1}(a) \geq 0$. If $\lambda_{k+1}(a)=0$, then $i(a)+\nu(a) \geq k+1$ and $i(b) \geq k+1$ and $\lambda_{k+1}(b)<0$. This is a contradiction. So $\lambda_{k+1}(a)>0$. Second, $\lambda_{k}(a) \leq 0$, we have $i(a)+\nu(a)=k$. Hence $i(b) \geq k$ and $\lambda_{k}(b)<0$. From the discussion above, we have $i(a)+\nu(a)=k=i(b)$.

(c) Case 1. $\lambda_{k}(a)=0$, and $\lambda_{k+1}(b)=0$.

Since $\lambda_{k}(a)=0$, then $i(a)+\nu(a) \geq k$. For $a<b$, we have $i(b) \geq i(a)+\nu(a) \geq$ $k$ and $\lambda_{k}(b)<0$. Together with $\lambda_{k+1}(b)=0$, we get $i(b)=k$ and $\nu(b) \neq 0$. Since 
$\lambda_{k+1}(b)=0$, then $i(b) \leq k$, and $i(a)+\nu(a) \leq k$. Hence $\lambda_{k+1}(a)>0$. Together with $\lambda_{k}(a)=0$, we obtain $i(a)+\nu(a)=k$ and $\nu(a) \neq 0$.

Case 2. If $i(a)+\nu(a)=k$ and $\nu(a) \neq 0$, then $\lambda_{k}(a)=0$. If $i(b)=k$ and $\nu(b) \neq 0$, then $\lambda_{k+1}(b)=0$.

Proposition 2.7. Let $a, b \in L^{\infty}(\Omega)$ satisfy $a<b$ and $i(a)+\nu(a)=i(b)$, $\nu(a) \neq 0, \nu(b) \neq 0$. Then the space $E$ has a decomposition:

$$
E=E^{-}(a) \oplus E^{0}(a) \oplus E^{0}(b) \oplus E^{+}(b) .
$$

Proof. First we show that $\left(E^{-}(a) \oplus E^{0}(a)\right) \cap\left(E^{0}(b) \oplus E^{+}(b)\right)=\emptyset$.

For any $u \in\left(E^{-}(a) \oplus E^{0}(a)\right) \cap\left(E^{0}(b) \oplus E^{+}(b)\right)$, we have $q_{a}(u, u) \leq 0$ and $q_{b}(u, u) \geq 0$. Furthermore, from $a<b$, we get $q_{a}(u, u)>q_{b}(u, u)$. This is a contradiction.

Now we only need to show that for any $u \in E, u=u_{1}+u_{2}$ with $u_{1} \in E^{-}(a) \oplus$ $E^{0}(a)$ and $u_{2} \in E^{0}(b) \oplus E^{-}(b)$. Let $\left\{e_{1}, \cdots e_{k}\right\}$ be a basis of $E^{-}(a) \oplus E^{0}(a)$ and $k=i(a)+\nu(a)=i(b)$. For each $e_{i}$, we have the decomposition $e_{i}=e_{i}^{-}+e_{i}^{*}$, $e_{i}^{-} \in E^{-}(b), e_{i}^{*} \in E^{0}(b) \oplus E^{-}(b)$. We claim that $\left\{e_{i}^{-}\right\}$are linear independent.

If not, there exists not all zero constants $c_{i}$ such that $\sum_{i}^{k} c_{i} e_{i}^{-}=0$. Let

$$
u=\sum_{i}^{k} c_{i} e_{i}=\sum_{i}^{k} c_{i} e_{i}^{*} \neq 0 .
$$

On the one hand,

$$
q_{b}(u, u)=q_{b}\left(\sum_{i}^{k} c_{i} e_{i}^{*}, \sum_{i}^{k} c_{i} e_{i}^{*}\right) \geq 0 ;
$$

on the other hand, $q_{a}(u, u) \leq 0$. This contradicts to $a<b$. Hence, $\left\{e_{1}^{-}, \ldots, e_{k}^{-}\right\}$ are linear independent in $E^{-}(b)$ and $\left\{e_{1}^{-}, \ldots, e_{k}^{-}\right\}$are a basis of $E^{-}(b)$.

For any $u \in E, u=u^{-}+u^{*}$ with $u^{-} \in E^{-}(b), u^{*} \in E^{0}(b) \oplus E^{+}(b)$,

$$
u=u^{-}+u^{*}=\sum_{i=1}^{k} c_{i} e_{i}^{-}+u^{*}=\sum_{i=1}^{k} c_{i} e_{i}+\left(u^{*}-\sum_{i}^{k} c_{i} e_{i}^{*}\right) .
$$

Here

$$
\sum_{i}^{k} c_{i} e_{i} \in E^{-}(a) \oplus E^{0}(a) \quad \text { and } \quad u^{*}-\sum_{i}^{k} c_{i} e_{i}^{*} \in E^{0}(b) \oplus E^{-}(b) .
$$

This completes the proof.

In order to prove Theorems 1.2 and 1.6, we introduce some lemmas about the existence and multiplicity of critical points in [1].

Lemma 2.8 ([1, Theorem 2.3]). Suppose that $f \in C^{\prime}(X, \mathbb{R})$ satisfies the following properties: 
(a) $f$ satisfies condition (C) in $[0,+\infty]$,

(b) there exist a closed subset $S \subset X$ and a Hilbert manifold $Q \subset X$ with boundary $\partial Q$ which fulfill the following properties:

(i) There exist two constants $\beta>\alpha \geq 0$ such that

$f(u) \leq \alpha, \quad$ for all $u \in \partial Q \quad$ and $\quad f(u) \geq \beta, \quad$ for all $u \in S$,

(ii) $S$ and $\partial Q$ link,

(iii) $\sup _{u \in Q} f(u)<+\infty$.

Then $f$ possesses a critical value $c \geq \beta$.

Here the condition $(\mathrm{C})$ means: For every sequence $\left\{u_{n}\right\} \subset X$ with

$$
I\left(u_{n}\right) \text { being bounded, } \quad(\|u\|+1) I^{\prime}\left(u_{n}\right) \rightarrow 0 \quad \text { as } n \rightarrow \infty
$$

possesses a convergent subsequence. This weaken condition was introduced by Cerami. This weakening of the Palais-Smale condition was proved to be essential in the study of variational problems in the strong resonance case in [1]. The condition (C) is actually sufficient to get a "deformation theorem".

Lemma 2.9 ([1, Lemma 2.4]). Suppose that $f \in C^{1}(H, \mathbb{R})$ satisfies the following properties:

(a) $f$ satisfies condition $(\mathrm{C})$ in $[0,+\infty]$ and $f(0) \geq 0$,

(b) there exist two closed subspaces $H^{+}, H^{-}$of $H$, with codim $H^{+}<+\infty$, and two constants $c_{\infty}>c_{0}>f(0)$ such that

(i) $f(u) \geq c_{0}$ for all $u \in S_{\rho} \cap H^{+}$,

(ii) $f(u)<c_{\infty}$ for all $u \in H^{-}$,

(c) $f$ is even.

Then, if $\operatorname{dim} H^{-} \geq \operatorname{codim} H^{+}, f$ possesses at least $m=\operatorname{dim} H^{-}-\operatorname{codim} H^{+}$distinct pairs of critical points whose corresponding critical values belong to $\left[c_{0}, c_{\infty}\right]$.

\section{Proofs of Theorems 1.2 and $\mathbf{1 . 6}$}

Proposition 3.1. Under the assumptions of Theorem 1.2, the functional defined in (1.3) satisfies the $(\mathrm{C})$ condition.

Proof. We will prove the proposition by several lemmas following the argument in [10] with modifications. Assume that $\left\{u_{n}\right\} \subset E$ satisfies $I\left(u_{n}\right)$ bounded and $\left(\left\|u_{n}\right\|+1\right) I^{\prime}\left(u_{n}\right) \rightarrow 0$. We first show that $u_{n}$ is bounded in $E$. We develop a contradiction argument, assume that $\left\|u_{n}\right\| \rightarrow \infty$ as $n \rightarrow \infty$. By $\left(\mathrm{f}_{1}\right)$, we obtain

$$
\left|f\left(x, u_{n}\right)\right| \leq c\left(1+\left|u_{n}\right|\right), \quad \text { a.e. on } \Omega \text {. }
$$

From (1.1),

$$
\int_{\Omega}\left(\Delta u_{n}, u_{n}\right) d x=\int_{\Omega} f\left(x, u_{n}\right) u_{n} d x+\int_{\Omega} f_{1}\left(x, u_{n}\right) u_{n} d x
$$


and

$$
\begin{aligned}
\left\|u_{n}\right\|^{2} & \leq \int_{\Omega}\left|f\left(x, u_{n}\right) u_{n}\right| d x+\int_{\Omega}|\widetilde{g}(x)|\left|u_{n}\right| d x \\
& \leq c \int_{\Omega}\left|u_{n}\right|+\left|u_{n}\right|^{2} d x+\left(\int_{\Omega}|\widetilde{g}(x)|^{2}\right)^{1 / 2}\left\|u_{n}\right\|_{2} \leq c\left\|u_{n}\right\|_{2}+c\left\|u_{n}\right\|_{2}^{2} .
\end{aligned}
$$

Hence, $\left\|u_{n}\right\| \leq c\left(\left\|u_{n}\right\|_{2}+1\right)$. Thus, $\left\|u_{n}\right\|_{2} \rightarrow \infty$ as $n \rightarrow \infty$. Let $z_{n}=u_{n} /\left\|u_{n}\right\|_{2}$. Since $E$ is compactly embedding in $L^{2}(\Omega)$, there exist $z \in E$ and a subsequence such that

$$
z_{n} \rightarrow z \text { weakly in } E, \quad \text { and } \quad z_{n} \rightarrow z \text { strongly in } L^{2}, \quad\left\|z_{n}\right\|_{2}=1 .
$$

By $\left(f_{2}\right)$, let

$$
c_{n}(x)=\frac{f\left(x, u_{n}(x)\right)}{u_{n}(x)} \quad \text { if }\left|u_{n}(x)\right| \geq r, \quad c_{n}(x)=a(x) \quad \text { if }\left|u_{n}(x)\right| \leq r .
$$

There is a $c \in L^{\infty}(\Omega)$ and $c_{n}(x) \rightarrow c(x)$ weakly in $L^{\infty}(\Omega)$ as $n \rightarrow \infty$ by going to subsequences if necessary. We have $a(x) \leq c(x) \leq b(x), x \in \Omega$. By (1.3), for any $v \in E$, we have

$$
\begin{aligned}
\left(I^{\prime}\left(u_{n}\right) \backslash\left\|u_{n}\right\|_{2}, v\right)= & \int_{\Omega} \nabla z_{n} \nabla v d x \\
& -\frac{1}{\left\|u_{n}\right\|_{2}} \int_{\Omega}\left(f\left(x, u_{n}\right), v\right) d x-\frac{1}{\left\|u_{n}\right\|_{2}} \int_{\Omega}\left(f_{1}\left(x, u_{n}\right), v\right) d x .
\end{aligned}
$$

Let $n \rightarrow \infty$. Standard computations show that

$$
\int_{\Omega} \nabla z \nabla v d x=\int_{\Omega} c(x) z v d x
$$

which implies $\nu(c) \neq 0$.

Case 1. $a<c$ and $c<b$.

From Proposition 2.5, we have $i(a)+\nu(a) \leq i(c)$ and $i(c)+\nu(c) \leq i(b)$ which contradicts to $i(a)+\nu(a)=i(b)$.

Claim. $c(x)=a(x)$ almost everywhere on $\Omega$.

We write

$$
I(u)=\frac{1}{2} \int_{\Omega}|\nabla u|^{2} d x-\frac{1}{2} \int_{\Omega} a(x) u^{2} d x-\int_{\Omega} G(x, u) d x-\int_{\Omega} F_{1}(x, u) d x,
$$

where $G(x, u)=\int_{0}^{u} g(x, t) d t, g(x, t)=f(x, t)-a(x) t$. Let $c_{n}^{1}(x)=c_{n}(x)-a(x)$. Then $c_{n}^{1}(x) \geq 0$ and $c_{n}^{1} \rightarrow 0$ weakly in $L^{\infty}(\Omega)$. From Proposition 2.1, $E^{-}(a)$ is finite dimensional, so $\left(-q_{a}\left(u^{-}, u^{-}\right)\right)^{1 / 2}$ is equivalent to $\|\cdot\|$.

$$
\begin{aligned}
& \text { For any } u=\sum_{j=k}^{\infty} c_{j} e_{j} \in E^{+}(a), k=i(a)+\nu(a)+1, \text { we have } \\
& q_{a}(u, u)=\sum_{j=k}^{\infty}\left(1-\lambda_{0} \mu_{j}(a)\right) c_{j}^{2} \geq\left(1-\lambda_{0} \mu_{k}(a)\right) \sum_{j=k}^{\infty} c_{j}^{2}=\left(1-\lambda_{0} \mu_{k}(a)\right)\|u\|^{2} .
\end{aligned}
$$


LEMma 3.2. There exists a constant $\delta>0$ such that

$$
\begin{array}{ll}
q_{a}(u, u) \leq-\delta\|u\|^{2}, & \text { for all } u \in E^{-}(a), \\
q_{a}(u, u) \geq \delta\|u\|^{2}, & \text { for all } u \in E^{+}(a) .
\end{array}
$$

From Proposition 2.1, $u_{n}$ has a decomposition:

$$
u_{n}=u_{n}^{+}+u_{n}^{0}+u_{n}^{-}, \quad u_{n}^{*} \in E^{*}(a), \quad *=+, 0,-.
$$

LEMMA 3.3. If $c(x)=a(x)$ almost everywhere on $\Omega$, we have $\left\|u_{n}^{+}\right\|^{2}+\left\|u_{n}^{-}\right\|^{2}$ is bounded.

Proof. Using (2.4), we have

$$
\left(\left\|u_{n}+1\right\|\right)\left\langle I^{\prime}\left(u_{n}\right), u_{n}^{+}-u_{n}^{0}-u_{n}^{-}\right\rangle=o(1)\left\|u_{n}^{+}-u_{n}^{0}-u_{n}^{-}\right\| .
$$

By Lemma 3.2, we have

$$
\begin{aligned}
& \left\langle I^{\prime}\left(u_{n}\right), u_{n}^{+}-u_{n}^{0}-u_{n}^{-}\right\rangle=q_{a}\left(u_{n}^{+}, u_{n}^{+}\right)-q_{a}\left(u_{n}^{-}, u_{n}^{-}\right) \\
& \quad-\int_{\Omega} g\left(x, u_{n}\right)\left(u_{n}^{+}-u_{n}^{0}-u_{n}^{-}\right) d x-\int_{\Omega} f_{1}\left(x, u_{n}\right)\left(u_{n}^{+}-u_{n}^{0}-u_{n}^{-}\right) d x \\
& \geq \delta\left(\left\|u_{n}^{+}\right\|^{2}+\left\|u_{n}^{-}\right\|^{2}\right)-\int_{\Omega} g\left(x, u_{n}\right)\left(u_{n}^{+}-u_{n}^{0}-u_{n}^{-}\right) d x \\
& \quad-\int_{\Omega} f_{1}\left(x, u_{n}\right)\left(u_{n}^{+}-u_{n}^{0}-u_{n}^{-}\right) d x .
\end{aligned}
$$

Together with (3.1), we have

(3.3) $\delta\left(\left\|u_{n}^{+}\right\|^{2}+\left\|u_{n}^{-}\right\|^{2}\right) \leq c \frac{\left\|u_{n}^{+}-u_{n}^{0}-u_{n}^{-}\right\|}{\left\|u_{n}\right\|+1}+\int_{\Omega} g\left(x, u_{n}\right)\left(u_{n}^{+}-u_{n}^{0}-u_{n}^{-}\right) d x$.

Since $u_{n}^{+}-u_{n}^{0}-u_{n}^{-}=2 u_{n}^{+}-u_{n}$, simple computation shows that

$$
\frac{\left\|u_{n}^{+}-u_{n}^{0}-u_{n}^{-}\right\|}{\left\|u_{n}\right\|+1} \leq c\left\|u_{n}^{+}\right\|+c, \quad \text { for } n \text { large enough. }
$$

Similar to [10, Lemma 2.4], we have

$$
\int_{\Omega} g\left(x, u_{n}\right)\left(u_{n}^{+}-u_{n}^{0}-u_{n}^{-}\right) d x \leq c\left\|u_{n}^{+}\right\|+\frac{\delta}{4}\left\|u_{n}^{+}\right\|^{2}+c,
$$

for $n$ large. Now we estimate the last part of (3.2), for any $M_{0}>0$,

$$
\begin{aligned}
\int_{\left|u_{n}\right| \leq M_{0}} & f_{1}\left(x, u_{n}\right)\left(u_{n}^{+}-u_{n}^{0}-u_{n}^{-}\right) d x \leq \int_{\left|u_{n}\right| \leq M_{0}} \tilde{g}(x)\left|2 u_{n}^{+}-u_{n}\right| d x \\
& \leq\left(\int_{\left|u_{n}\right| \leq M_{0}} \widetilde{g}^{2}(x)\right)^{1 / 2}\left(\int_{\left|u_{n}\right| \leq M_{0}}\left|2 u_{n}^{+}-u_{n}\right|^{2}\right)^{1 / 2} \leq c\left\|u_{n}^{+}\right\|+c .
\end{aligned}
$$


By the Sobolev inequality for $n \leq 4, E \hookrightarrow L_{4}$,

$$
\begin{gathered}
\int_{\left|u_{n}\right| \geq M_{0}} f_{1}\left(x, u_{n}\right)\left(u_{n}^{+}-u_{n}^{0}-u_{n}^{-}\right) d x \leq \int_{\left|u_{n}\right| \geq M_{0}} \frac{f_{1}\left(x, u_{n}\right)}{u_{n}}\left(u_{n}^{+2}-\left(u_{n}^{0}+u_{n}^{-}\right)^{2}\right) d x \\
\leq \frac{1}{M_{0}} \int_{\left|u_{n}\right| \geq M_{0}} \widetilde{g}(x) u_{n}^{+2} d x \leq \frac{1}{M_{0}} c\left\|u_{n}^{+}\right\|_{4}^{2} \leq \frac{1}{M_{0}} c\left\|u_{n}^{+}\right\|^{2} \leq \frac{\delta}{4}\left\|u_{n}^{+}\right\|^{2}
\end{gathered}
$$

for large constant $M_{0}$. Hence,

$$
\int_{\Omega} f_{1}\left(x, u_{n}\right)\left(u_{n}^{+}-u_{n}^{0}-u_{n}^{-}\right) d x \leq c\left\|u_{n}^{+}\right\|+\frac{\delta}{4}\left\|u_{n}^{+}\right\|^{2}+c .
$$

From (3.3)-(3.6), we have $\left\|u_{n}^{+}\right\|^{2}+\left\|u_{n}^{-}\right\|^{2}$ is bounded.

LEMMA 3.4. If $\left\|u_{n}^{+}\right\|^{2}+\left\|u_{n}^{-}\right\|^{2}$ is bounded, then $I\left(u_{n}\right) \rightarrow-\infty$ as $n \rightarrow \infty$.

Proof. Since $\left\|u_{n}^{+}\right\|^{2}+\left\|u_{n}^{-}\right\|^{2}$ is bounded, we get

$$
\begin{aligned}
I\left(u_{n}\right)= & \frac{1}{2} \int_{\Omega}\left|\nabla u_{n}\right|^{2} d x-\frac{1}{2} \int_{\Omega} a(x) u_{n}^{2} d x-\int_{\Omega} G\left(x, u_{n}\right) d x-\int_{\Omega} F_{1}\left(x, u_{n}\right) d x \\
\leq & c+\int_{\Omega}\left[G\left(x, \frac{u_{n}^{0}}{2}\right)-G\left(x, u_{n}\right)\right] d x+\int_{\Omega}\left[F_{1}\left(x, \frac{u_{n}^{0}}{2}\right)-F\left(x, u_{n}\right)\right] d x \\
& -\int_{\Omega} G\left(x, \frac{u_{n}^{0}}{2}\right)+F_{1}\left(x, \frac{u_{n}^{0}}{2}\right) d x .
\end{aligned}
$$

From [10, Lemma 2.5], we obtain

$$
\int_{\Omega}\left[G\left(x, \frac{u_{n}^{0}}{2}\right)-G\left(x, u_{n}\right)\right] d x \leq c .
$$

In order to prove the boundness of $\int_{\Omega}\left[F_{1}\left(x, u_{n}^{0} / 2\right)-F_{1}\left(x, u_{n}\right)\right] d x$, we follow the proof of (3.7) closely. By the mean value theorem, we have

$\int_{\Omega}\left[F_{1}\left(x, \frac{u_{n}^{0}}{2}\right)-F_{1}\left(x, u_{n}\right)\right] d x=\int_{\Omega} \int_{0}^{1} f_{1}\left(x, t \frac{u_{n}^{0}}{2}+(1-t) u_{n}\right)\left(\frac{u_{n}^{0}}{2}-u_{n}\right) d t d x$.

Assume that $h_{n}=t u_{n}^{0} / 2+(1-t) u_{n}$, by $\left|f_{1}(x, u)\right| \leq \widetilde{g}(x)$, one has

$$
\begin{aligned}
\int_{\left|h_{n}\right| \leq M_{0}}\left[F_{1}\left(x, \frac{u_{n}^{0}}{2}\right)\right. & \left.-F_{1}\left(x, u_{n}\right)\right] d x \leq \int_{\left|h_{n}\right| \leq M_{0}} \widetilde{g}(x)\left|\frac{u_{n}^{0}}{2}-u_{n}\right| d t d x \\
= & \int_{\left|h_{n}\right| \leq M_{0}} \widetilde{g}(x)\left|\frac{1}{t-2} h_{n}+\frac{1}{t-2}\left(u_{n}^{0}-u_{n}\right)\right| d t d x \\
\leq & c\left(\int_{\left|h_{n}\right| \leq M_{0}}\left|h_{n}+\left(u_{n}^{-}+u_{n}^{+}\right)\right|^{2}\right)^{1 / 2} \leq c
\end{aligned}
$$


On the other hand,

$$
\begin{gathered}
\int_{\left|h_{n}\right| \geq M_{0}}\left[F_{1}\left(x, \frac{u_{n}^{0}}{2}\right)-F_{1}\left(x, u_{n}\right)\right] d x=\int_{\left|h_{n}\right| \geq M_{0}} \int_{0}^{1} f_{1}\left(x, h_{n}\right)\left(\frac{u_{n}^{0}}{2}-u_{n}\right) d t d x \\
=\int_{\left|h_{n}\right| \geq M_{0}} \int_{0}^{1} \frac{f_{1}\left(x, h_{n}\right)}{h_{n}}\left[t\left(\frac{u_{n}^{0}}{2}-u_{n}\right)^{2}+\left(\frac{u_{n}^{0}}{2}-u_{n}\right) u_{n}\right] d t d x \\
\leq \int_{\left|h_{n}\right| \geq M_{0}} \frac{\tilde{g}(x)}{M_{0}}\left[\left(\frac{u_{n}^{0}}{2}-u_{n}\right)^{2}+\left(\frac{u_{n}^{0}}{2}-u_{n}\right) u_{n}\right] d x
\end{gathered}
$$

By the elementary inequality,

$$
\left(\frac{u_{n}^{0}}{2}-u_{n}\right)^{2}+\left(\frac{u_{n}^{0}}{2}-u_{n}\right) u_{n} \leq\left(u_{n}^{+}+u_{n}^{-}\right)^{2} .
$$

We have

$$
\begin{aligned}
\int_{\left|h_{n}\right| \geq M_{0}}\left[F_{1}\left(x, \frac{u_{n}^{0}}{2}\right)-F_{1}\left(x, u_{n}\right)\right] d x & \leq \int_{\left|h_{n}\right| \geq M_{0}} \frac{\widetilde{g}(x)}{M_{0}}\left(u_{n}^{+}+u_{n}^{-}\right)^{2} d x \\
& \leq c\left\|u_{n}^{+}+u_{n}^{-}\right\|_{L^{4}}^{2} \leq c\left\|u_{n}^{+}+u_{n}^{-}\right\|^{2} \leq c .
\end{aligned}
$$

This together with the condition $\left(\mathrm{f}_{3}^{+}\right)$gives $I\left(u_{n}\right) \rightarrow-\infty$, as $n \rightarrow \infty$.

Case 3. $c(x)=b(x)$ almost everywhere on $\Omega$.

This case is similar to Case 2. We have the following lemma.

Lemma 3.5. If $c(x)=b(x)$ almost everywhere on $\Omega$, then $\left\|u_{n}^{+}\right\|^{2}+\left\|u_{n}^{-}\right\|^{2}$ is bounded and $I\left(u_{n}\right) \rightarrow+\infty$ as $n \rightarrow \infty$.

Lemmas 3.4, 3.5 contradict to condition (2.4), which implies Cases 2 and 3 are also impossible. Hence the proof of Proposition 3.1 is completed.

Proof of Theorem 1.2. We apply Lemma 2.11 to the functional $I$ defined by (1.3). Let $X_{1}=E^{-}(a) \oplus E^{0}(a), X_{2}=E^{0}(b) \oplus E^{+}(b)$. Then $E=X_{1} \oplus X_{2}$ and $X_{1}$ is finite dimensional.

First, we have $I(u) \rightarrow-\infty$ as $\|u\| \rightarrow \infty, u \in X_{1}$.

If not, there exists a sequence $u_{n}=u_{n}^{-}+u_{n}^{0} \in E^{-}(a) \oplus E^{0}(a)$ such that $\|u\| \rightarrow \infty$ and $I(u) \geq c$.

$$
\begin{aligned}
I\left(u_{n}\right) & =\frac{1}{2} \int_{\Omega}\left|\nabla u_{n}\right|^{2} d x-\frac{1}{2} \int_{\Omega} a(x) u_{n}^{2} d x-\int_{\Omega} G\left(x, u_{n}\right) d x-\int_{\Omega} F_{1}\left(x, u_{n}\right) d x \\
& =q_{a}\left(u_{n}^{-}, u_{n}^{-}\right)-\int_{\Omega} G\left(x, u_{n}\right) d x \int_{\Omega}-F_{1}\left(x, u_{n}\right) d x \leq-\delta\left\|u_{n}^{-}\right\|^{2}+c .
\end{aligned}
$$

Therefore, $\left\|u_{n}^{-}\right\|^{2}$ is bounded. And by Lemma 3.4 , we obtain $I(u) \rightarrow-\infty$, as $n \rightarrow \infty$. This is a contradiction.

By Lemma 3.5, similar to the discussion above, we get $I\left(u_{n}\right) \rightarrow+\infty$ as as $\|u\| \rightarrow \infty, u \in X_{2}$.

Let $Q=B_{R} \cap X_{1}, S=X_{2}$. Then $\partial Q$ and $S$ link. Thus it is easy to verify the assumptions of Lemma 2.11 . 
Proof of TheOrem 1.6. In order to prove Theorem 1.6, we only need to check that the assumptions of Lemma 2.9 are satisfied. Such work will be done in the following Lemmas 3.6 and 3.7.

LEMMA 3.6. The assumption (b)(i) of Lemma 2.9 is valid, where $f$ is defined as (1.3) and $\operatorname{codim} H^{+}=i\left(a_{1}\right)+\nu\left(a_{1}\right)$.

Proof. Let $\varepsilon>0$ small enough and set $4<r<2 n /(n-2), n<4$. Since $\left(\mathrm{f}_{1}\right)$ and $\left(\mathrm{f}_{4}\right)$ hold, there exist $\delta_{2}>\delta_{1}>0$ and $M>0$ for the above $\varepsilon$ such that

$$
\begin{array}{cl}
F(x, u)+F_{1}(x, u) \leq \frac{1}{2}\left(a_{1}(x)+\varepsilon\right)|u|^{2}, & \text { for all }|u| \leq \delta_{1}, x \in \bar{\Omega}, \\
F(x, u)+F_{1}(x, u) \leq M|u|^{2} \leq \frac{M}{\delta_{1}^{r-2}}|u|^{r}, & \delta_{1} \leq|u| \leq \delta_{2}, x \in \bar{\Omega}, \\
F(x, u) \leq \frac{1}{2}(b(x)+\varepsilon)|u|^{2} \leq \frac{1}{2 \delta_{2}^{r-2}}\left(\|b(x)\|_{\infty}+\varepsilon\right)|u|^{r}, & \text { for all }|u| \geq \delta_{2}, x \in \bar{\Omega} .
\end{array}
$$

Furthermore,

$$
\int_{|u| \geq \delta_{2}} F_{1}(x, u) d x \leq\left(\int_{|u| \geq \delta_{2}} \widetilde{g}^{2}(x) d x\right)^{1 / 2}\left(\int_{|u| \geq \delta_{2}}|u|^{2} d x\right)^{1 / 2} \leq \frac{c}{\delta_{2}^{r-2}}\|u\|_{L^{r}}^{r / 2} .
$$

Set

$$
A=\max \left\{\frac{1}{2 \delta_{2}^{r-2}}\left(\|b(x)\|_{\infty}+\varepsilon\right), \frac{M}{\delta_{1}^{r-2}}\right\}>0 .
$$

Together with (1.3), we obtain

$$
\begin{aligned}
I(u) & \geq \frac{1}{2} \int_{\Omega}|\nabla u|^{2} d x-\int_{\Omega} \frac{1}{2}\left(a_{1}(x)+\varepsilon\right)|u|^{2}+A|u|^{r} d x-\frac{c}{\delta_{2}^{r-2}}\|u\|_{L^{r}}^{r / 2} \\
& =\frac{1}{2} q_{a_{1}+\varepsilon}(u, u)-A\|u\|_{L^{r}}^{r}-\frac{c}{\delta_{2}^{r-2}}\|u\|_{L^{r}}^{r / 2} .
\end{aligned}
$$

Let $H^{+}=E^{+}\left(a_{1}+\varepsilon\right)$. Then $\operatorname{codim} H^{+}=i\left(a_{1}+\varepsilon\right)+\nu\left(a_{1}+\varepsilon\right)$. For any $u \in H^{+}$, we have

$$
I(u) \geq \frac{1}{2}\left(1-\lambda_{0} \mu_{k}\left(a_{1}+\varepsilon\right)\right)\|u\|^{2}-A c\|u\|^{r}-\frac{c}{\delta_{2}^{r-2}}\|u\|^{r / 2} .
$$

Here $\mu_{k}\left(a_{1}+\varepsilon\right)$ is defined in Proposition 2.1 and $k=i\left(a_{1}+\varepsilon\right)+\nu\left(a_{1}+\varepsilon\right)+1$. Since $1-\lambda_{0} \mu_{k}\left(a_{1}+\varepsilon\right)>0$, let $\rho>0$ small enough such that

$$
I(u) \geq c_{0}=\frac{1}{2}\left(1-\lambda_{0} \mu_{k}\left(a_{1}+\varepsilon\right)\right) \rho^{2}-A c \rho^{r}-\frac{c}{\delta_{2}^{r-2}} \rho^{r / 2}>0 .
$$

Furthermore, from Proposition 2.5, pick $\varepsilon$ small enough, we have

$$
i\left(a_{1}+\varepsilon\right)+\nu\left(a_{1}+\varepsilon\right)=i\left(a_{1}\right)+\nu\left(a_{1}\right) .
$$

So we have $I(u) \geq c_{0}$, for all $u \in S_{\rho} \cap H^{+}$and codim $H^{+}=i\left(a_{1}\right)+\nu\left(a_{1}\right)$. The proof is completed. 
Lemma 3.7. The assumption (b)(ii) of Lemma 2.9 is also satisfied and

$$
\operatorname{dim} H^{-}=i(a)-\nu(a)
$$

Proof. $\left(f_{1}\right)$ holds. Let

$$
J(u)=\frac{1}{2} \int_{\Omega}|\nabla u|^{2} d x-\int_{\Omega} F(x, u) d x .
$$

For any $\varepsilon>0$, there exists a constant $M$ such that

$$
J(u) \leq \frac{1}{2} \int_{\Omega}|\nabla u|^{2} d x-\frac{1}{2} \int_{\Omega}(a(x)-\varepsilon)|u|^{2} d x+M=\frac{1}{2} q_{a-\varepsilon}(u, u)+M,
$$

for all $u \in E$. Recall that $\left|f_{1}(x, u)\right| \leq \widetilde{g}(x)$, where $\widetilde{g}(x) \in L^{2}$, and the embedding theorem, we get

$$
\left|\int_{\Omega} F_{1}(x, u) d x\right| \leq \int_{\Omega} \widetilde{g}(x)|u| d x \leq c\|u\| .
$$

Combining (3.7) and (3.8), we obtain

$$
I(u)=J(u)-\int_{\Omega} F_{1}(x, u) d x \leq \frac{1}{2} q_{a-\varepsilon}(u, u)+c\|u\|+M .
$$

Let $H^{-}=E^{-}(a-\varepsilon)$. Then $\operatorname{dim} H^{-}=i(a-\varepsilon)$. From Lemma 3.2, there exists a constant $\delta_{0}$, such that

$$
\frac{1}{2} q_{a-\varepsilon}(u, u) \leq-\delta_{0}\|u\|^{2} \text { for any } u \in H^{-} .
$$

Thus, $I(u) \leq-\delta_{0}\|u\|^{2}+c\|u\|+M<+\infty$. Also from Proposition 2.5, pick $\varepsilon$ small enough, we have $i(a-\varepsilon)=i(a)-\nu(a)$.

Proof of Corollaries 1.3 And 1.7. For $f_{1}(x, u)=0$, the proof of Corollary 1.3 and 1.7 can be easily deduced from the proof of Theorems 1.2 and 1.6 for any $n$, respectively.

\section{REFERENCES}

[1] P. Bartolo, V. Benci And D. Fortunato, Abstract critical point theorems and applications to some nonlinear problem with "strong" resonance at infinity, Nonlinear Anal. 7 (1983), 981-1012.

[2] K.C. Chang, Critical point theory and its application, Shanghai Sci. Tech. Press, Shanghai, 1986. (in Chinese).

[3] _ Infinite dimentional Morse theory and multiple solution problems, Birkhäuser. Basel, 1993.

[4] C. Conley And E. Zehnder, Morse-type index theory for flows and periodic solutions for Hamiltonian equations, Comm. Pure Appl. Math. 37 (1984), 207-253.

[5] Y. Dong, On equivalent conditions for the solvability of equation $\left(p(t) x^{\prime}\right)^{\prime}+f(t, x)=$ $h(t)$ satisfying linear boundary conditions with $f$ restricted by linear growth conditions, J. Math. Anal. Appl. 245 (2000), 204-220.

[6] _ Index theory, nontrivial solutions, and asymptotically linear second-order Hamiltonian systems, J. Differential Equations 214 (2005), 233-255. 
[7] _ Maslov type index theory for linear Hamiltonian systems with Bolza boundary value conditions and multiple solutions for nonlinear Hamiltonian systems, Pacific J. Math. 221 (2005), 253-280.

[8] _ Index theory for linear selfadjoint operator equations and nontrivial solutions for asymptotically linear operator equations, Calc. Var. 38 (2010), 75-109.

[9] C. FABry And A. Fonda, Periodic solutions of nonlinear differential equations with double resonance, Ann. Mat. Pura Appl. 157 (1990), 99-116.

[10] M. Jiang And M. Sun, A semilinear elliptic equation with double resonance, Acta Math. Sin. (Engl. Ser.) 27 (2011), 1233-1246.

[11] E. LANDESMAN AND A.C. LAzer, Nonlinear perturbations of lineae eigenvalues problem at resonance, J. Math. Mech. 19 (1970), 609-623.

[12] K. LI, Multiple periodic solutions for asymptotically linear Duffing equations with resonance, J. Math. Anal. Appl. 378 (2011), 657-666.

[13] _ Multiple solutions for an asymptotically linear Duffing equation with Newmann boundary value conditions, Nonlinear Anal. 74 (2011), 2819-2830.

[14] Y. Long, Maslov-type index theory, degenerate critical points, and asymptotically linear Hamiltonian systems, Sci. China Ser. A 33 (1990), 1409-1419.

[15] _ A Maslov-type index theory for symplectic paths, Topol. Methods Nonlinear Anal. 10 (1997), 47-78.

[16] J. Mawhin, M. Willem, Critical point theory and Hamiltonian systems, Springer, New York, 1989.

[17] J.B. Su, Semilinear elliptic boundary value problems with double resonance between teo consecutive eigenvalues, Nonlinear Anal. 48 (2002), 881-895.

[18] J. Su AND L. ZhaO, Multiple periodic solutions of ordinary differential equations with double resonance, Nonlinear Anal. 70 (2009), 1520-1527.

Manuscript received September 1, 2012

YuAN SHan

Department of Mathematics

Nanjing University

Nanjing, Jiangsu 210093, P.R. CHINA

E-mail address: shannjnu@gmail.com

KEQIANG Li

Department of Mathematics

Henan Normal University

Henan, P.R. CHINA

E-mail address: likeqiang000@sina.com 